\title{
Le pouvoir en échec : fiction mémorielle et reconstruction littéraire dans Adiós Muchachos de Sergio Ramírez
}

Jean-Henri Madeleine

\section{CpenEdition}

Journals

Édition électronique

URL : https://journals.openedition.org/cher/12059

DOI : $10.4000 /$ cher. 12059

ISSN : 2803-5992

Éditeur

Presses universitaires de Strasbourg

Édition imprimée

Date de publication : 30 juin 2012

Pagination : 51-62

ISBN : 978-2-35410-046-9

ISSN : 1968-035X

Référence électronique

Jean-Henri Madeleine, "Le pouvoir en échec : fiction mémorielle et reconstruction littéraire dans Adiós Muchachos de Sergio Ramírez », reCHERches [En ligne], 8 | 2012, mis en ligne le 21 février 2022, consulté le 23 février 2022. URL : http://journals.openedition.org/cher/12059 ; DOI : https://doi.org/ 10.4000/cher.12059

\section{cc) (i) $\$($ )}

Ce(tte) œuvre est mise à disposition selon les termes de la Licence Creative Commons Attribution -

Pas d'Utilisation Commerciale - Partage dans les Mêmes Conditions 4.0 International. 


\title{
Le pouvoir en échec: fiction mémorielle et reconstruction littéraire dans Adiós Muchachos de Sergio Ramírez
}

\author{
Jean-Henri Madeleine \\ Université Paris-Est
}

$\mathrm{D}$ ans la littérature latino-américaine $\mathrm{du} \mathrm{XX}^{\mathrm{e}}$ siècle, les déclinaisons fictionnelles de l'échec du pouvoir politique sont nombreuses. Qu'elles remontent au traumatisme originel de la Conquête, insistent sur la faillite $\mathrm{du}$ projet d'unification et de refondation bolivarien, ou mettent l'accent sur la déréliction constitutive à tout pouvoir personnel, ces œuvres ont très souvent en commun une vision en quelque sorte "pendulaire» de l'histoire des sociétés latino-américaines, qui oscillent entre "civilisation» et "barbarie», ou entre l'effort des Latino-américains pour sortir de leur «mismidad» (Picotti 1991: 194) et le retour aux tares de l'«ExtrêmeOccident» (Rouquié 1987: 12).

Ainsi les écrivains latino-américains dans leur majorité ne prennent-ils pas en compte la distinction classique entre l'échec individuel (qui renvoie à la béance entre l'intention et la réalisation de celle-ci et est donc lié à un sujet) et l'insuccès, collectif (en ce qu'il renvoie à des causes extérieures au sujet lui-même). Dans ce contexte, les régimes révolutionnaires constituent des matériaux littéraires privilégiés, au regard de la thématique de l'échec. Ils offrent en effet la possibilité d'évoquer, à l'intersection de l'individuel et du collectif, les processus de flux et de reflux des intentions de reconstruction d'une humanité nouvelle par de nouveaux hommes et le retour à des schémas de comportements antérieurs. 
Ainsi après le Mexique, après Cuba, le Nicaragua sandiniste occupe-til une place spécifique dans la littérature latino-américaine du $\mathrm{XX}^{\mathrm{e}}$ siècle. Avec quelques singularités profondes cependant: la période révolutionnaire est relativement brève ${ }^{1}$ (dix années au total, entre 1979 et 1989), le régime semble très rapidement et constamment menacé (en raison de la guerre civile soutenue par l'administration Reagan, mais également de la faillite du "projet économique»), et surtout elle s'achève par des élections démocratiques et l'éviction ${ }^{2}$ des dirigeants sandinistes du pouvoir. La conjonction de la radicalité et de la brièveté du projet de transformation tranchent ainsi fortement avec les précédents cubains et mexicains. De plus, la convergence de la chute du Mur de Berlin et de celle du régime sandiniste marquent également, dans l'imaginaire collectif latino-américain et occidental, la fin des projets utopiques nationaux et continentaux nés de la révolution cubaine et l'entrée dans ce que l'on appellera la «postmodernité».

C’est pourquoi, en dépit de leur forte hétérogénéité esthétique, les mouvements littéraires surgis dans les deux dernières décennies en Amérique latine, du Crack mexicain au McOndo chilien possèdent en commun ce même refus des «métarécits» révolutionnaires et des "romans nationaux». Ce refus, qu'il prenne la forme d'une "dénationalisation» de l'entreprise littéraire ou d'une critique plus culturaliste de "l'homme latino-américain » participe donc d'une littérature du désenchantement en réaction au réalisme magique et à ses diverses déclinaisons, tout comme aux nouveaux codes esthétiques nés de la diffusion des moyens de communication de masse. Le refus de l'utopie politique s'accompagne donc souvent d'une césure dans l'ordre esthétique, qui intègre de façon presque systématique une composante générationnelle. Cette césure est naturellement graduelle, et il apparaît utile, dans cette perspective, d'analyser la façon dont elle se cristallise pour les auteurs qui ont commencé à écrire dans les années 1960

1 Cette brièveté est une des raisons pour lesquelles le terme «revolución» est utilisé chez de nombreux auteurs - dont Sergio Ramírez - pour désigner tant la période de conquête du pouvoir que celle de l'hégémonie du parti révolutionnaire. Nous utiliserons donc par commodité le terme "révolution» afin de désigner ces deux étapes, tout en ayant à l'esprit la différence entre ces temporalités définie par Pierre Vayssière, «l'enracinement des vainqueurs et la pérennisation des nouveaux maitres, habituellement justifiés par une prétendue "consolidation» de la révolution, ne semblent guère compatibles avec l'authenticité de l'esprit de révolte ni avec la créativité de l'engagement révolutionnaire, par essence fugitif» (Vayssière 1991: 18).

2 Celle-ci est bien entendu très relative: le FSLN garde le contrôle de l'armée (Humberto Ortega, frère de Daniel Ortega, président entre 1984 et 1989, dirige celle-ci), ainsi que des forces de police et de nombreux relais dans les administrations publiques. 
et 1970. L'œuvre que l'on étudiera ici très brièvement, Adiós Muchachos de l'écrivain nicaraguayen Sergio Ramírez semble, à ce titre, particulièrement représentative. En effet, elle traite de l'histoire de l'ensemble du processus révolutionnaire, des années de lutte durant les années 1970 à la réalisation du projet révolutionnaire dans la décennie des années 1980.

\section{Au cœur du pouvoir, au cœur de l'échec}

Né en 1942 à Masaya, Sergio Ramírez, juriste de formation, s'essaie très rapidement à la poésie et au conte dans les diverses publications ${ }^{3}$ surgies grâce aux lois d'autonomie universitaire octroyées par le pouvoir dans les années 1960. Son implication dans les révoltes étudiantes le conduit à se rapprocher de l'opposition anti-somoziste, dont il sera l'une des figures centrales puisqu'il assurera la direction du "grupo de los doce $»^{4}$. La victoire de 1979, à laquelle il participe activement, l'oblige à mettre entre parenthèses son œuvre littéraire. Sa place au sein du nouveau régime devient en effet de plus en plus centrale. A l'exception de José Vasconcelos au Mexique, il est très certainement l'un des hommes de lettres latino-américains ayant exercé les fonctions politiques nationales les plus importantes au $\mathrm{XX}^{\mathrm{e}}$ siècle.

Ce pouvoir est multiple: culturel et symbolique tout d'abord; directeur de la maison d'édition «Nueva Nicaragua», il est également, comme on l'a dit précédemment, au cœur de la construction de ce «lieu de mémoire» qu'est devenu Sandino pour tous les Nicaraguayens; politique ensuite: membre de la junte du gouvernement de reconstruction nationale (JGRN), puis secrétaire général de la présidence de la république, il est vice-président de la république entre 1984 et 1989. Il sera, après la défaite électorale de 1989, chef du groupe parlementaire sandiniste à l'assemblée nationale jusqu'en 1995.

L'auteur de Adiós Muchachos est donc plus qu'un simple "compagnon de route», c'est un homme politique qui s'est inscrit au cœur d'un mouvement d'opposition à la dictature, puis d'un projet de refondation de la société et de l'homme sur des bases révolutionnaires marxistes. Ce sont donc ces années de lutte contre la dictature, puis d'exercice du pouvoir qu'il relate dans Adiós muchachos, dont la première édition date de 1999, c'est-à-dire dix ans après

3 Notamment la revue Ventana née du Frente Ventana créé par l'auteur avec Fernando Gordillo.

4 Le "grupo de los doce» était une réunion de personnalités supposément indépendantes de tout parti, provenant de la société civile censées incarner une opposition à Somoza plus "policée», au regard de l'opinion publique nationale et surtout internationale, que celle représentée par le FSLN. 
la fin de cette expérience révolutionnaire et quatre ans après avoir quitté le FSLN ${ }^{5}$, alors que sa vie politique est, semble-t-il du moins, terminée.

Ainsi l'échec que relate ce récit dont le sous-titre est Una memoria de la revolución sandinista semble-t-il, au premier abord, total. Échec individuel de l'intention d'inscrire un sens dans une trajectoire existentielle, mais aussi échec collectif de la praxis révolutionnaire, enfin deuil définitif du principe d'espérance contenu dans le "grand récit» marxiste qui fondait cette expérience. Dans cette perspective, la question posée sera double: quelle organisation et quelles stratégies narratives sont mises en œuvres afin de rendre compte de cet échec comme faillite de l'action et de la raison; corrélativement, quelle place cette œuvre prend-elle dans le rapport à l'échec qu'entretiennent les écrivains centraméricains des vingt dernières années?

\section{Entre tentation historiciste et pulsion épique}

Ainsi que nous l'indiquions, le sous-titre de Adiós Muchachos est Una memoria de la revolución sandinista. Le seuil du texte joue donc ici un rôle programmatique fondamental: l'utilisation du terme "memoria» et de l'indéfini viennent d'emblée placer l'œuvre de Sergio Ramírez dans le genre de la littérature de témoignage, née d'ailleurs en partie à l'occasion des conflits internes en Amérique centrale. Ce choix de l'inscription dans la «mémoire» relève, semble-t-il, d’une stratégie discursive spécifique. Sergio Ramírez, exclu de l'appareil du parti, ne peut commettre, ainsi que l'ont fait des responsables politiques du FSLN', un ouvrage relatant une histoire «canonique» de la révolution. Il choisit donc une position en quelque sorte intermédiaire: une mémoire dont les lacunes, les béances justifient de façon proleptique ce que le récit de son implication dans la révolution pourrait avoir d'incomplet, voire de frustrant pour le lecteur. Position non dépourvue d'ambiguïtés et de tensions entre le personnage public devenu témoin et l'auteur, entre la mémoire et l'histoire.

La dimension personnelle que le terme de mémoire induit n'est en effet repérable qu’à de rares moments dans le texte. De fait, la part du «moi» est évacuée en quelque sorte avec le premier chapitre, intitulé «Confesión de parte». Celui-ci opère la jonction métonymique du «roman familial» et du «roman national», à travers l'évocation des grandes étapes de la vie de ses enfants (Sergio, María et Dorel), depuis leur naissance au

5 Les raisons de ce départ sont explicitées dans le récit de façon assez incomplète.

6 Notamment Humberto Ortega. 
milieu des années 1960 jusqu'à la fin des années 1990, au moment de sa dernière campagne présidentielle: la croisade nationale d'alphabétisation, l'engagement politique, le service militaire, la transition et l'adaptation à la paix. La perspective adoptée de manière beaucoup plus fréquente par l'auteur est celle que Ranke indiquait comme la plus appropriée à l'historien: celle de l'homme d'État. Tout d'abord à travers le dispositif para-textuel: une «cronología básica» en fin d'ouvrage, notamment, qui ne retient que les avancées sociales et le conflit avec l'administration Reagan, au détriment de la guerre civile et du processus de monopolisation du pouvoir par le FSLN. Cette analyse "surplombante» et pour tout dire déterministe de l'échec de la révolution en tant que régime se décline en trois axes thématiques majeurs: les relations internationales, les rapports avec l'Eglise, la politique macroéconomique. Ces axes «traversent» l'ensemble du texte mais l'auteur y consacre aussi trois chapitres spécifiques. Les problèmes géopolitiques sont analysés dans le chapitre VII, intitulé «El destino manifiesto». Les biais idéologiques des dirigeants du FSLN tout comme ceux des membres de l'administration Carter puis Reagan sont évoqués sans fard:

[...] la proclamación de nuestra soberanía sólo podía hacerse en contra de Estados Unidos, y nuestro nacionalismo nacía de esa contradicción. La nación había estado confiscada, y para Nicaragua, como país pequeño, la razón misma de su existencia se ligaba a su independencia [...] Lo repetíamos con la retórica más virulenta en las plazas públicas, en las arengas radiales, en los editoriales de Barricada, y todos los análisis de la realidad política se basaban en ese presupuesto (138).

De même, dans le chapitre IX, «El paraíso en la tierra », Sergio Ramírez propose une explication de la question très complexe des désaccords entre l'Eglise catholique et le parti, avec en point d'orgue la visite du Pape au Nicaragua. Les raisons à la fois structurelles (la mise en place d'une nouvelle «religiosité » révolutionnaire, la présence de prêtres favorables à la théologie de la libération) et conjoncturelles (les événements en Pologne, la coïncidence d'une opération particulièrement meurtrière de la Contra avec la visite du Pape) de l'échec de cette visite et de l'opposition toujours plus marquée de l'Eglise officielle au régime sandiniste y sont analysées de façon à la fois neutre et circonstanciée.

C’est enfin, essentiellement dans le chapitre XI, «Ríos de leche y miel», les errements de la politique macroéconomique qui sont évoqués de façon à nouveau très détaillée. La guerre n'est pas, comme dans la plupart des récits des dirigeants du FSLN, invoquée pour justifier la faillite du projet économique. C'est d'abord l'échec majeur de la réforme agraire qui est exposée 
sans fard, et, plus précisément, le refus d'accorder aux paysans la pleine et entière propriété de leur terre. La construction de structures de production collectives identifiables aux «sovkhozes" soviétiques est rétrospectivement considérée comme une erreur majeure. Le second motif d'explication de l'échec économique invoqué est relatif aux erreurs ainsi qu'à l'inefficacité structurelle de la planification dans un contexte politique d'hégémonie du FSLN, d'absence d'unité de commandement réel de celui-ci, dans l'ordre économique tout du moins. C'est donc la structure du pouvoir en elle-même qui apparaît comme la cause de l'effondrement du régime. Après l'échec de la réforme agraire, après la critique d'un désir de planification plaqué sur un appareil étatique clivé en autant de chapelles, c'est le fondement théorique de la révolution qui est finalement analysé et dénoncé par Sergio Ramírez. La possession des moyens de production ainsi que le contrôle social exercé par le parti ne pouvaient conduire au développement, car l'accumulation de capital ne pouvait être réalisée par l'État. L'un des points cardinaux du «matérialisme scientifique» est ici récusé de façon explicite:

Pero pienso que aun sin guerra, las sustancias filosóficas del modelo que buscábamos aplicar habrían conducido de todos modos a un colapso económico, a no ser por una revolución pacífica del sistema hacia una economía mixta real, lo que a su vez hubiera demandado una mayor apertura política [...] el modelo de acumulación, basado en la idea del Estado dueño, no fue viable desde el principio (240-241).

On mesure avec cette dernière citation l'ampleur de l'aggiornamento doctrinal opéré par Sergio Ramírez. L'étatisation des moyens de production est considérée comme une erreur fondamentale. Plus encore: le processus d'accumulation est lui aussi dénoncé. Or c'est bien, dans la théorie marxiste, ce processus d'accumulation qui permet l'émergence de la bourgeoisie en tant que classe possédante. Sergio Ramírez esquisse les implications politiques que la reconnaissance de ce "point aveugle» de la théorie marxiste aurait entraîné : une «révolution pacifique» qui aurait eu pour but la création d'une «économie mixte réelle» et finalement «l'ouverture politique». De fait, le seul moyen d'éviter la faillite du régime sur le plan économique aurait abouti à... la fin du régime mais dans sa dimension politique, ou son évolution vers la social-démocratie. On le voit, la «mémoire» de Sergio Ramírez apparaît très fréquemment comme celle d'un intellectuel dont l'aspiration à la rationalisation, sinon à la rationalité historique affleure régulièrement dans 
le texte ${ }^{7}$. Mais cette position n'est cependant pas univoque et ne le conduit pas à opérer un décentrement complet et à se situer dans une perspective que l'on pourrait qualifier en quelque sorte de «post-politique», c'est-à-dire dans laquelle l'action comme moyen de transformation du collectif disparaît. En effet, symétriquement à ce discours de rationalisation historique de l'échec, apparaît dans Adiós Muchachos ce que l'on pourrait qualifier de tentation «épique » ou "paléo-épique». Toute l'œuvre est traversée de ces oscillations entre deux pulsations discursives: celle de l'argumentation, de la justification des décisions des dirigeants de l'État-FSLN auxquels l'auteur s'associe et celle de la célébration lyrique des «héros révolutionnaires» dont Sergio Ramírez se fait le porte-parole, comme dans nombre de ses autres œuvres, qu'elles soient ou non proprement romanesques.

Cette pulsation épique est repérable, tout d'abord, dans les «seuils» du texte. Ainsi l'œuvre est-elle dédiée à Dora María Téllez. Cette dernière a atteint rapidement un statut «iconique» en participant à la prise de l'assemblée nationale le 22 juillet 1978 - autre «lieu de mémoire» de l'identité sandiniste - en tant que "comandante dos». Ministre de la Santé pendant la décade sandiniste, elle participe, avec l'auteur, à la tentative de rénovation du FSLN dans la première moitié des années 90 , puis à la création du $\mathrm{MRS}^{8}$, parti qui tente de rompre avec l'hégémonie du dirigeant historique Daniel Ortega et ses pratiques politiques. Cette double référence, à l'une des figures les plus respectées de l'histoire contemporaine du Nicaragua, mais aussi à l'un des moments les plus «héroïques» de la lutte contre la dictature, inscrit ainsi d'emblée le récit dans une continuité éthique au regard du titre. La loyauté au regard de l'impulsion révolutionnaire dans sa signification première de combat contre l'ordre injuste est préservée. Ainsi s'opère une "suturation» de la mémoire nationale: par-delà les années de guerre et de dictature du FSLN, les exempla individuels de l'opposition à l'arbitraire du pouvoir restent.

Après cette dédicace une citation vient renforcer ce schéma de reconstruction implicite de la mémoire: «La canción de gesta fue un periódico que llevó el viento» qui est un vers du poème Oráculo sobre Managua de Ernesto Cardenal. La référence au poète qui, l'un des premiers,

7 Cette «position d'autorité » est renforcée par les citations régulières d'ouvrages d'historiens et de politistes (le plus souvent anglo-saxons) de la période.

8 Le «Movimiento de Renovación Sandinista» est créé officiellement et de façon symbolique le 18 mai 1995, jour du centième anniversaire de la naissance d'Augusto César Sandino. 
tenta de réincorporer la figure de Sandino dans l'imaginaire national", prêtre adepte de la théologie de la libération, créateur de la communauté de Solentiname, puis ministre de la culture pendant les années 1980, agit de la même façon que celle de Dora María Téllez. De façon identique, le vers cité inscrit explicitement le récit dans l'«épopée», une épopée qui suppose un monde «inaccessible et clos» (Bakhtine, 1978: 461), mais dont le «substrat héroïque» irrigue encore l'imaginaire collectif et l'écriture elle-même.

La construction de l'œuvre elle-même présente une forte cohérence avec cette "programmation para-textuelle». En effet, sur les treize chapitres de Adiós Muchachos, seuls quatre sont consacrés aux années d'exercice du pouvoir dans leur ensemble ${ }^{10}$. C'est donc la période antérieure à la révolution qui est massivement évoquée, celle de la lutte clandestine, puis ouverte contre la dictature somoziste. Ce biais temporel aboutit à un fort clivage dans l'ordre des thèmes traités mais aussi dans celui des techniques d'écriture. L'évocation des années de dictature et de la victoire articule l'évocation fréquemment lyrique des figures sacrificielles du mouvement révolutionnaire (Sandino ${ }^{11}$, le père Gaspar García Laviana ${ }^{12}$, etc.) et l'évocation souvent parodique et carnavalesque des partisans de la dictature et du dictateur lui-même. La structure de l'œuvre repose entièrement sur cette dissociation entre les temps de la pureté et ceux de la déréliction liée à l'exercice du pouvoir, sur cette césure entre la mystique de la lutte révolutionnaire et l'échec politique d'un régime hégémonique. Les titres des chapitres indiquent clairement cette pulsation: du chapitre II «Vivir como los santos» et III «La edad de la inocencia», on passe au V «La edad de la malicia» puis au IX «El paraíso en la tierra» et au XI «Ríos de leche y miel» qui est à interpréter de façon parodique puisqu'il décrit, ainsi que nous l'avons indiqué, la faillite du projet économique. Adiós muchachos apparaît donc comme une œuvre «en

9 Notamment dans son recueil de poèmes Hora 0, publié en 1960.

10 Outre le premier chapitre, «Confesión de parte», le chapitre V «La edad de la malicia» qui traite notamment de la monopolisation du pouvoir par le FSLN dans les temps qui succèdent à la chute de Somoza; le chapitre VII «El destino manifiesto » auquel nous avons fait allusion auparavant et qui a trait aux relations avec les Etats-Unis et aux problèmes géopolitiques posés par la révolution sandiniste; le chapitre IX qui, comme nous en avons fait mention, relate les relations avec le Vatican, enfin le chapitre XI, «Ríos de leche y miel» aussi évoqué, sur la politique macroéconomique.

11 Carlos Fonseca (1936-1976) est l'un des fondateurs et dirigeants principaux du FSLN. Il meurt au combat alors que son autorité effective sur le mouvement avait très fortement diminué.

12 Prêtre d'origine asturienne qui décidera de rejoindre la guérilla. Il meurt lui aussi au combat, en 1978. 
tension ", dans laquelle l'analyse puis le témoignage, la célébration lyrique et la critique maîtrisée alternent dans une organisation temporelle qui n'est ni totalement chronologique, ni purement soumise à l'arbitraire du souvenir.

\section{La « ruse de la raison » et la confluence des mémoires}

Il apparaît évident que l'analyse des errements et erreurs politiques des dix années du pouvoir sandiniste est menée avec une certaine rigueur, dans Adiós Muchachos. La réforme agraire, les relations avec les EtatsUnis, l'Eglise, la continuité de pratiques caudillistes sont évoquées de manière précise. Néanmoins il est tout aussi évident que d'autres facteurs fondamentaux de cet échec ne sont pas évoqués. Le point nodal de ces failles de la mémoire concerne la guerre elle-même, dans sa relation avec la réforme agraire. Les erreurs de celle-ci sont, comme on l'a dit, reconnues de façon explicite. Cependant l'incompréhension de la révolte paysanne est repérable à de nombreuses reprises dans le texte. Ainsi dans le chapitre 11 évoque-t-il un vieux paysan lié à la Contra que l'on force, lors d'un meeting électoral en 1984, à rendre ses armes devant lui:

No sólo por novelista era yo un intelectual, igual a los demás que vestían uniformes de comandantes, y también decían discursos y teorizaban. Todos, desde arriba, pensábamos la revolución en términos de teoría o de ideal, y esa concepción mental trataba de ser aplicada o impuesta a la sociedad, y a gente de carne y hueso como el campesino humilde y acobardado que me entregaba el rifle. Le proponíamos el viaje incomprensible desde lo primitivo a lo moderno, pero él se negaba y había tomado un arma para oponerse (230).

Dans le même chapitre, les mêmes arguments sont peu ou prou utilisés concernant les populations de la partie orientale du Nicaragua. Cette séquence appelle quelques commentaires. Elle développe la césure classique entre les idéaux et l'action, en ce qui concerne les responsables politiques. La connaissance directe que Sergio Ramírez pouvait avoir du système cubain, mais aussi des démocraties populaires en obère cependant fortement la valeur. De même, son autodéfinition en tant que membre d'un groupe dirigeant constitué d'intellectuels minore-t-il sa position à l'intérieur du régime. Il est, à l'époque, candidat à la vice-présidence de la république et au cœur du pouvoir depuis 1979: cette brusque "révélation» du hiatus entre la politique mise en œuvre et ses effets semble pour le moins tardive. Mais c'est certainement la référence à la dyade "primitif/ moderne» qui s'avère ici éclairante. Elle s'offre en effet comme une déclinaison de l'antithèse "civilisation/barbarie» qui informe, depuis le XIX ${ }^{e}$ siècle, 
le champ intellectuel et politique latino-américain. Ainsi l'échec de la révolution est-il imputable non seulement au modèle de transformation choisi, mais également à une résistance, une incompréhension d'ordre social, voire ontologique. L'insuccès de la révolution serait donc lié à un échec inhérent aux élites latino-américaines - et surtout aux intellectuels des années soixante, hérauts et guides des sans-voix de l'Histoire - qui consiste à faire emprunter aux masses paysannes le «chemin» qui les conduirait de l'archaïsme à la modernité, c'est-à-dire à les «civiliser».

Ce pessimisme radical quant à l'idée et la possibilité du progrès n'est cependant pas systématique dans Adiós Muchachos. On serait tenté de dire que c'est le contraire qui se produit: on perçoit dans toute l'œuvre, en filigrane, la foi dans la raison et la politique. La preuve la plus évidente en est la thèse centrale de la différence entre «la táctica revolucionaria» qui conduit les sandinistes à s'allier avec les autres segments de l'opposition anti-somozistes, puis à célébrer des élections présidentielles en 1984, et la «estrategia a largo plazo » qui était, dans l'esprit des commandants sandinistes, l'établissement d'un régime socialiste. Cette tactique du compromis momentané, de la mise entre parenthèses du «projet totalitaire» était bien, selon l'auteur au cœur du «tercérisme», la troisième tendance du FSLN:

El nuestro fue un régimen muy democrático, en un sentido nuevo, y muy autoritario, en un sentido viejo. Pasados los años, lo que se llamó el proyecto táctico terminó imponiéndose, como ya dije, y la democracia, ya sin apellidos, ni burguesa, ni proletaria, vino a ser el fruto más visible de la revolución. La gran paradoja fue que, al fin y al cabo, el sandinismo dejó en herencia lo que no se propuso: la democracia, y no pudo heredar lo que se propuso : el fin del atraso, la pobreza y la marginación» (112-115).

La vision qui sous-tend cette explication générale des années d'hégémonie révolutionnaire reste finalement très hégélienne: la révolution et ses erreurs apparaissent, dix ans après, comme une "ruse de la raison», qui valide, a posteriori, son action. La démocratie est un résultat paradoxal de la révolution. Le retournement dialectique n'est cependant pas total: la misère sociale continue d'exister, elle est la dimension de l'échec du sandinisme comme régime. Mais la démocratie (favorisée par des pratiques électorales plus justes, des forces de police et une armée plus professionnelles et plus neutres, affirme-t-il par ailleurs) est née de l'action des tenants de la tendance tercériste tels que lui qui, pendant les dix années de pouvoir du FSLN, ont privilégié la tactique à l'idéologie, la négociation à l'opposition radicale. Ainsi l'insuccès politique est-il une étape vers l'entrée du Nicaragua dans la modernité. 


\section{Conclusion : réparation mémorielle et dépassement de l'échec}

Cette résistance au pessimisme post-révolutionnaire est réaffirmée à la fin de l'ouvrage, dans l'épilogue. Celui-ci organise métonymiquement la «réparation des mémoires ». Celle des victimes anonymes de la révolution, dont les orphelins, adoptés par des volontaires espagnols, viennent le rencontrer lors d'une séance de dédicace de son ouvrage précédent à Saragosse. Celle des militantes du FSLN, par le biais de la fille de l'une d'elles, Idania Fernández, morte avant la victoire et ayant laissé une fillette de 4 ans, Claudia. Celle-ci, devenue adulte, lui demandera à plusieurs reprises de le rencontrer. Cette rencontre est décrite dans le dernier paragraphe de l'ouvrage, et rentre en confluence avec celle des orphelins:

Esa vez de mi encuentro con Claudia, ya en la calle, prontos a despedirnos, le pregunté, un tanto cohibido, si pensaba que el sacrificio de su madre había valido la pena. Yo hubiera hecho lo mismo, me dijo sin pensarlo dos veces, las manos en los bolsillos del abrigo de lana... Y copio el resto de sus palabras, que anoté al volver a mi apartamento: ella no dio su vida en vano. Lo hizo por impulso del corazón, por su amor sin egoísmo, y puso el bienestar de los demás por encima de su propia vida. Y no importan los resultados, importa su ideal. Sobre todo, agregó, en este tiempo sin ideales, y me sonrió, muy serena. Se alejó hacia la boca del metro y se volvió para decirme adiós con la mano, sonriendo otra vez. Y yo entonces pensé: suerte que la revolución sigue siendo un niño que viene de la mano por el pasillo de una librería, se te acerca y te sonríe con la sonrisa de Claudia, que es la misma sonrisa de Idania (294-295).

A travers cette métaphore «germinale» se trouvent légitimées simultanément l'expérience révolutionnaire collective et celle des individus, mais plus encore celle de l'écriture - et de l'écrivain - (voir « copio », " anoté », «librería») qui permet la jonction du passé et du présent, et la justification du premier par le second... La césure avec les écrivains nicaraguayens et centraméricains de la génération post-révolutionnaire est patente: faut-il y voir l'attachement persistant de Sergio Ramírez aux valeurs de la modernité, et particulièrement la résistance au deuil définitif du rôle des intellectuels comme relais et fondateurs de la mémoire nationale?

\section{Bibliographie}

Aguirre Erick, 2005, Subversión de la memoria, Tendencias en la narrativa centroamericana de postguerra, Managua, ANE-CNE. 
Aínsa, Fernando, 1994, La reescritura de la historia en la nueva narrativa latinoamericana, San José, Universidad de Costa Rica.

Arellano, Jorge Eduardo, 1997, Literatura nicaragüense, Managua, Distribuidora cultural.

Arellano, Jorge Eduardo, 2003, Literatura centroamericana. Diccionario de autores contemporáneos. Fuentes para su estudio, Managua, Fundación Vida.

Bakhtine, Mikhail, 1978, Esthétique et théorie du roman, Paris, Gallimard.

Beverley, John et Zimmerman, Marc, 1990, Literature and Politics in the Central American Revolutions, Austin, University of Texas Press.

Delgado Aburto, Leonel, 2002, Márgenes recorridos. Apuntes sobre procesos culturales y literatura nicaragüense del siglo XX, Managua, IHNCA-UCA.

Hartog, François, 2003, Régimes d'historicité. Présentisme et expériences du temps, Paris, Seuil.

Lyotard, Jean-François, 1979, La condition post-moderne, Paris, Minuit.

Martí y Puig, Salvador et Close, David (eds.), 2009, Nicaragua y el FSLN 1979-2009. ¿Qué queda de la revolución?, Barcelona, edicions bellaterra.

Midence, Carlos, 2008, La Invención de Nicaragua. Letra y Polis en la conformación de la nación, Managua, Amerisque.

Morales, Moisés Hassan, 2009, La maldición del Güegüense, Managua, PAVSA.

Pérez-Baltodano, Andrés, 2003, Entre el Estado Conquistador y el Estado Nación. Providencialismo, pensamiento político y estructuras de poder en el desarrollo histórico de Nicaragua, Managua, IHNCA-UCA.

Picotti, Dina, 1991, El descubrimiento de América y la otredad de las culturas. 1492-1992. A los 50 años del choque de dos mundos. Balance y prospectiva, Buenos Aires, RundiNuskin editor.

Rouquié, Alain, 1991, Amérique latine. Introduction à l'Extrême-Occident, Paris, Seuil.

Vayssière, Pierre, 2001, Les révolutions d'Amérique latine, Paris, Seuil. 